\title{
Resultado imediato do tratamento endovascular com stents paralelos do aneurisma roto justarrenal
}

\author{
Immediate outcome of endovascular treatment of ruptured juxtarenal aneurysm with \\ parallel stents
}

\begin{abstract}
Claudia Guimarães Agle (D), César Amorim Pacheco Neves², Flávia Dórea Carneiro Abbehusen³,
\end{abstract} Tainã Lemos Andrade 3 , Filinto Marques de Cerqueira Neto ${ }^{4}$, Dejean Sampaio Amorim Filho ${ }^{4}$

\section{Resumo}

A ruptura do aneurisma de aorta abdominal é um evento com alta mortalidade, e o seu tratamento nesses casos é uma emergência médica. O tratamento endovascular desses aneurismas tem se estabelecido como uma alternativa minimamente invasiva à cirurgia aberta clássica, tornando-se a opção de primeira escolha. Contudo, 20 a $50 \%$ dos pacientes portadores de aneurisma de aorta abdominal não apresentam anatomia favorável para o tratamento endovascular devido à presença de colo curto ou pelo acometimento de ramos viscerais pelo aneurisma. Relatamos um caso de uma paciente de 70 anos submetida à correção endovascular de aneurisma roto justarrenal com implante de stents paralelos para as renais (técnica de chaminé). São apresentados dados clínicos e detalhes do procedimento. O sucesso técnico foi obtido e não houve relato de complicações pós-operatórias.
\end{abstract}

Palavras-chave: aneurisma roto; procedimentos endovasculares; aneurisma da aorta abdominal; stents; ruptura.

\begin{abstract}
Rupture of an abdominal aortic aneurysm is an event with a high mortality rate and treatment is a medical emergency. Endovascular treatment of these aneurysms has become established as a minimally invasive alternative to classical open surgery and is now the first-choice option. However, 20 to 50\% of patients with abdominal aortic aneurysms do not have anatomy favorable for endovascular treatment because of a short aneurysm neck or because visceral branches are involved by the aneurysm. We report the case of a 70-year-old patient who underwent endovascular repair of a ruptured juxtarenal aneurysm with deployment of parallel stents in the renal arteries (in a chimney technique). Clinical data and details of the procedure are reported. Technical success was achieved and there were no postoperative complications.
\end{abstract}

Keywords: ruptured aneurysm; endovascular procedures; abdominal aortic aneurysm; stents; rupture.

Como citar: Agle CG, Neves CAP, Abbehusen FDC, Andrade TL, Cerqueira Neto FM, Amorim Filho DS. Resultado imediato do tratamento endovascular com stents paralelos do aneurisma roto justarrenal. J Vasc Bras. 2021;20:e20200120. https://doi.org/10.1590/1677-5449.200120 


\section{INTRODUÇÃO}

$\mathrm{O}$ aneurisma de aorta abdominal (AAA) constitui um desafio para o cirurgião vascular, especialmente quando se estende para os vasos viscerais (aneurismas complexos). A ruptura do AAA é um evento com alta mortalidade, e a cirurgia ou o tratamento endovascular nesses casos é uma emergência médica. $\mathrm{O}$ risco de ruptura do aneurisma é proporcional ao seu tamanho, sendo que aneurismas medindo menos de 5,4 cm têm uma taxa de ruptura anual de aproximadamente $1 \%$, enquanto aqueles maiores que $7,0 \mathrm{~cm}$ de diâmetro têm uma taxa de ruptura anual de $32,5 \%{ }^{1}$. Esse risco é ainda quatro vezes maior em mulheres do que em homens $^{2}$. O tratamento endovascular (TE) desses aneurismas tem se estabelecido como uma alternativa minimamente invasiva à cirurgia aberta clássica, tornando-se a opção de primeira escolha. Essa evolução é baseada principalmente nos ensaios multicêntricos randomizados EVAR-1 (Endovascular Aneurysm Repair 1), DREAM (Diabetes Reduction Assessment with ramipril and rosiglitazone Medication) e OVER (Open versus Endovascular Repair) ${ }^{3-6}$. Contudo, 20 a $50 \%$ dos pacientes portadores de AAA não apresentam anatomia favorável para o TE devido à presença de colo curto ou pelo acometimento de ramos viscerais pelo aneurisma, e sabe-se que AAAs com comprimento do colo proximal menor que 10 milímetros estão associados a um risco aumentado de reintervenção e óbito $^{7}$. Entre as opções para esses casos, são factíveis as técnicas com endopróteses fenestradas, ramificadas, modificadas pelo cirurgião e stents paralelos. A técnica com stents paralelos (chaminé) baseia-se na colocação de stents nos vasos viscerais em paralelo ao corpo principal da endoprótese ${ }^{8}$. Apresentamos aqui um caso de ruptura de AAA justarrenal tratado através da técnica endovascular com implante de stents paralelos para as renais em um serviço particular na cidade de Salvador, BA.

O estudo foi aprovado pelo Comitê de Ética da Fundação Bahiana de Cardiologia (FBC) de Salvador, BA, Brasil (Parecer n 4.341.672).

\section{PARTE I - SITUAÇÃO CLÍNICA}

Paciente do sexo feminino, 70 anos. Tabagista, hipertensa, foi encaminhada ao serviço com dor intensa em flanco direito e pelve. Foi feita suspeita diagnóstica de nefrolitíase e solicitada tomografia com contraste. Esta identificou aneurisma fusiforme de $8 \mathrm{~cm}$ com sinais sugestivos de rotura (Figura 1) e justarrenal (Figuras 2 e 3 ).

Diante desse quadro, algumas opções terapêuticas foram aventadas:

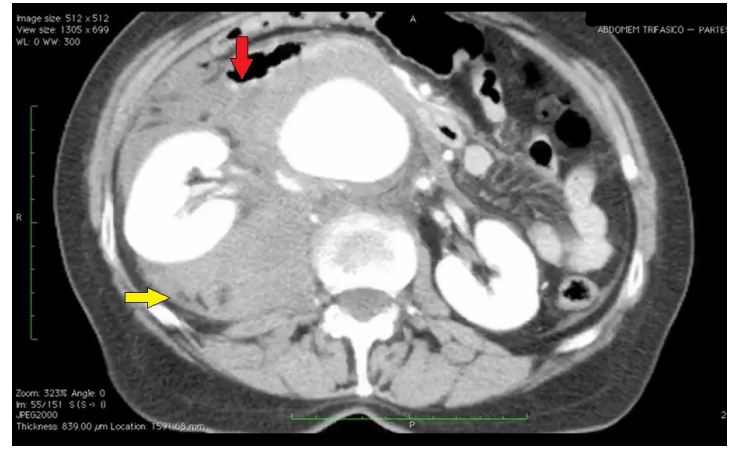

Figura 1. Angiotomografia demonstrando aneurisma da aorta abdominal (AAA) roto com hematoma retroperitoneal à direita. Seta vermelha: ruptura do aneurisma (descontinuidade da parede do AAA). Seta amarela: hematoma retroperitoneal.

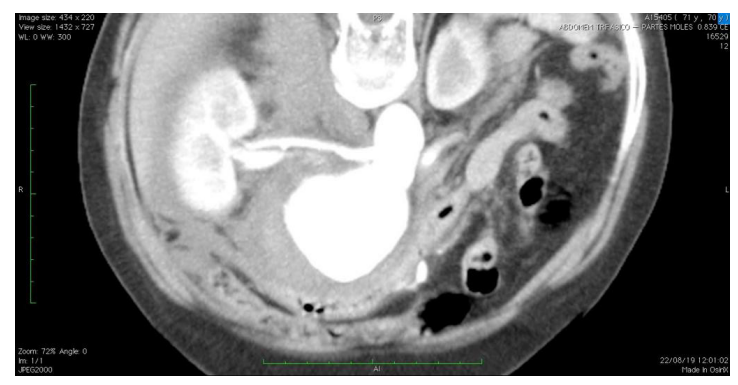

Figura 2. Angiotomografia demonstrando aneurisma da aorta abdominal justarrenal.

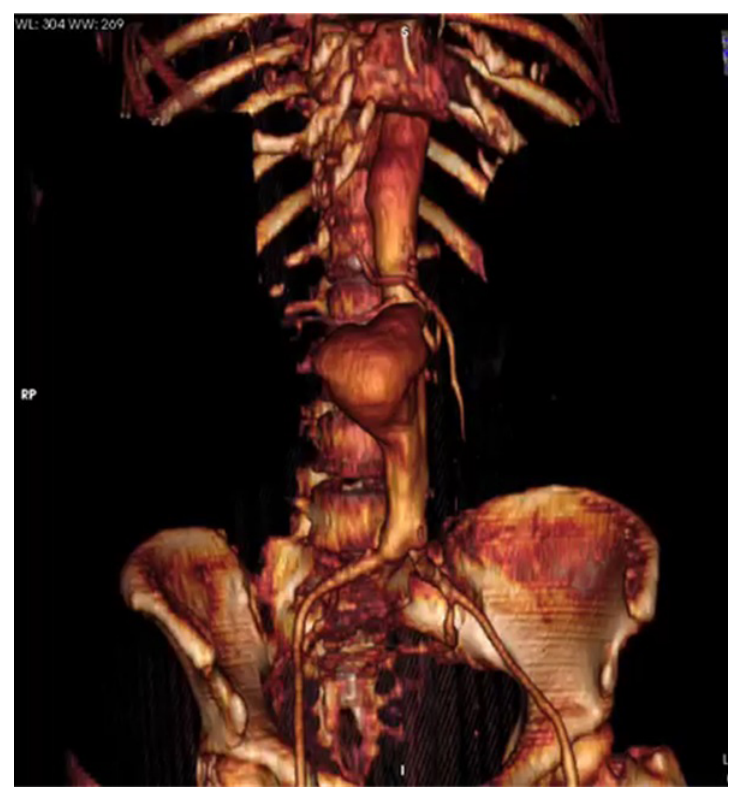

Figura 3. Reconstrução tomográfica demonstrando aneurisma da aorta abdominal justarrenal.

1- Procedimento endovascular (endoprótese fenestrada, ramificada, modificada pelo cirurgião ou técnica com stents paralelos);

2- Cirurgia aberta convencional. 


\section{PARTE II - O QUE FOI FEITO}

A paciente foi submetida à anestesia geral, e o tratamento endovascular foi realizado. Quatro acessos foram necessários para o procedimento: dissecção de artéria femoral comum bilateral e punção das artérias braquiais. Foi inserido na artéria femoral direita um introdutor $12 \mathrm{~F}$, na artéria femoral esquerda, um introdutor $16 \mathrm{~F}$ e nas artérias braquiais, um introdutor 7F longo. Pela artéria femoral esquerda, foi posicionado o corpo da endoprótese (Figura 4) GORE C $3{ }^{\circledR}$ (W. L. Gore \& Associates, Inc., Delaware, EUA) (26 x 14 x 18) próximo à artéria mesentérica superior. Pelas artérias braquiais, foram cateterizadas as artérias renais e posicionados os stents autoexpansíveis recobertos VIABAHN 6 × 50 (Figura 5). O corpo da endoprótese foi liberado até a perna contralateral, e, em seguida, foram liberados os stents das artérias renais (Figura 6). Após isso, pela artéria femoral direita, a extensão contralateral da endoprótese ( $16 \mathrm{x}$ 14 x 12) foi implantada. Foi realizada aortografia de controle, demostrando endoprótese sem vazamentos e com perviedade de artérias renais (Figuras 7 e 8). Não foi utilizado balão de acomodação no seguimento proximal da endoprótese. A paciente permaneceu na unidade de terapia intensiva (UTI) por 24 horas, tendo alta hospitalar após 48 horas. Realizou-se tomografia de controle com 30 dias, que demonstrou endoprótese e stents de artérias renais bem-posicionados e sem sinais de fratura (Figura 9).

\section{DISCUSSÃO}

O tempo de intervenção para o AAA roto afeta diretamente os resultados. Estes pacientes devem

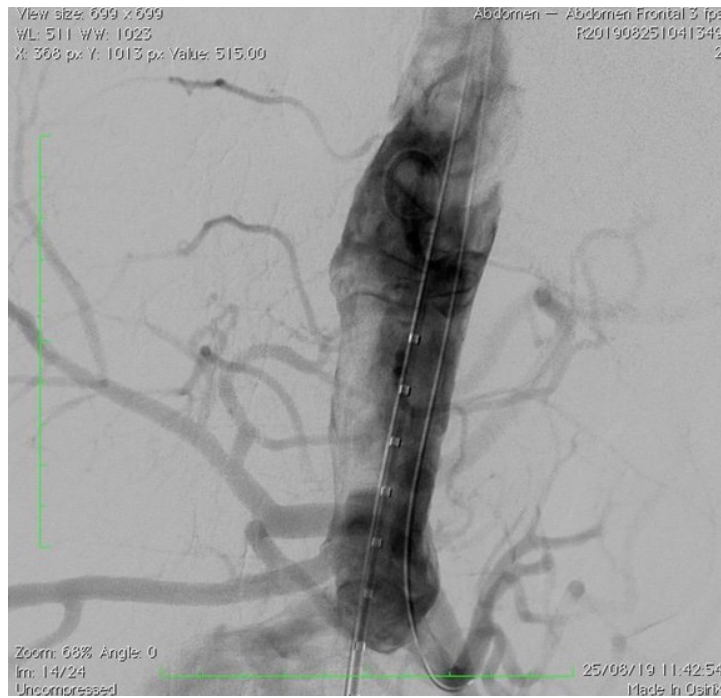

Figura 4. Corpo da endoprótese posicionada na aorta proximal, com cateterização seletiva da renal esquerda. ter seu diagnóstico identificado o mais precocemente possível, com rápido encaminhamento a uma unidade hospitalar cuja estrutura permita o tratamento adequado da patologia. Segundo a diretriz publicada em 2018 pela Society for Vascular Surgery (SVS), a meta de intervalo porta-balão deve ser menor que 90 minutos, com o tempo zero sendo definido como o tempo do primeiro contato médico, e a intervenção definida a partir do acesso arterial inicial e colocação de um balão de oclusão aórtica. Apesar da indicação, cumprir o atendimento nesse curto espaço de tempo ainda é desafiador. Em 2004, o Registro Nacional de Dados Cardiovasculares dos Estados Unidos informou que,

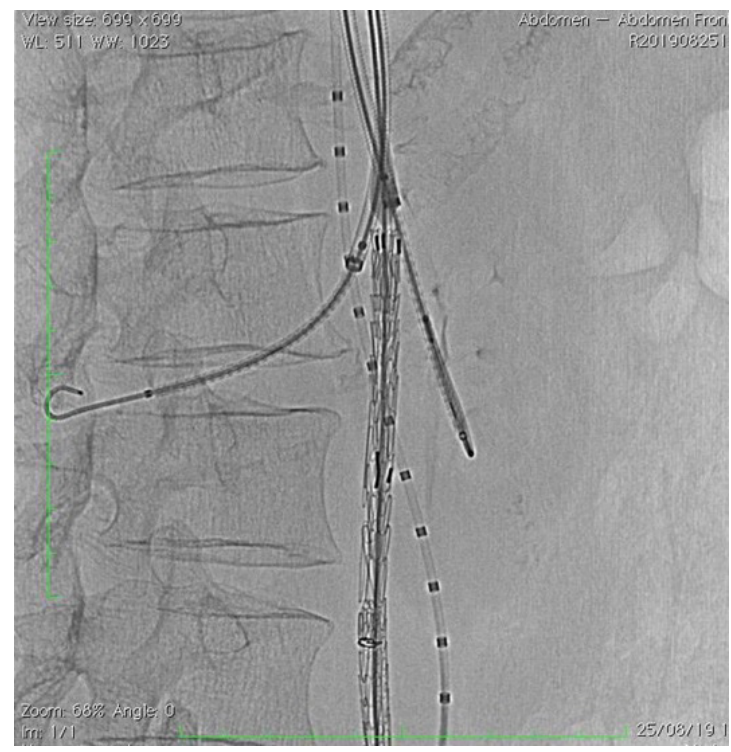

Figura 5. Stents autoexpansíveis VIABAHN posicionados nas artérias renais.

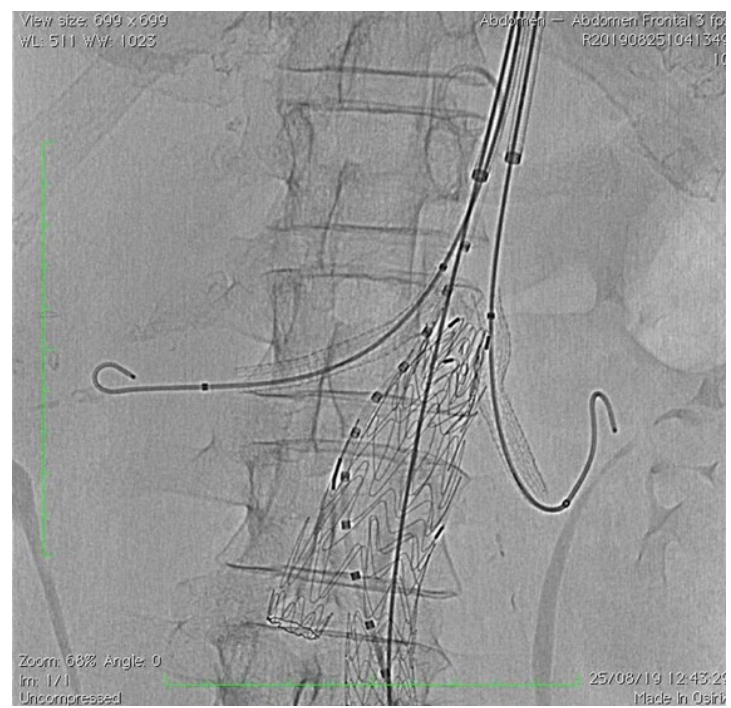

Figura 6. Corpo da endoprótese e stents autoexpansíveis das renais liberados. 


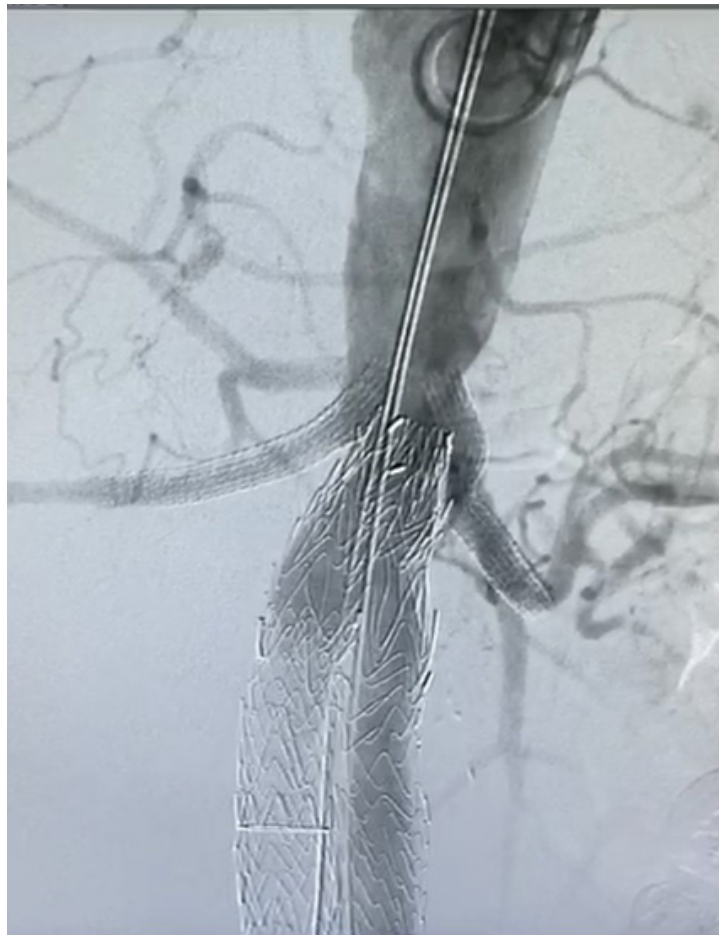

Figura 7. Aortografia de controle demonstrando seguimento proximal da endoprótese sem vazamentos e stents de artérias renais bem-posicionados.

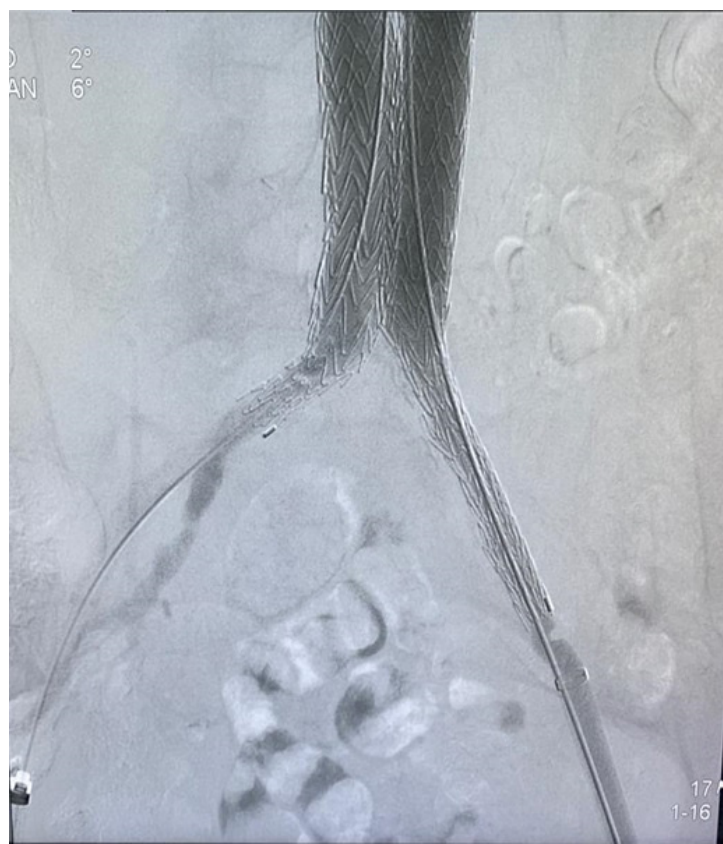

Figura 8. Aortografia de controle demonstrando seguimento distal da endoprótese sem vazamentos.

para pacientes que necessitaram de transferência inter-hospitalar, apenas $8,6 \%$ tinham cumprido o tempo porta-balão menor que 90 minutos, com um tempo médio de 152 minutos 9 .

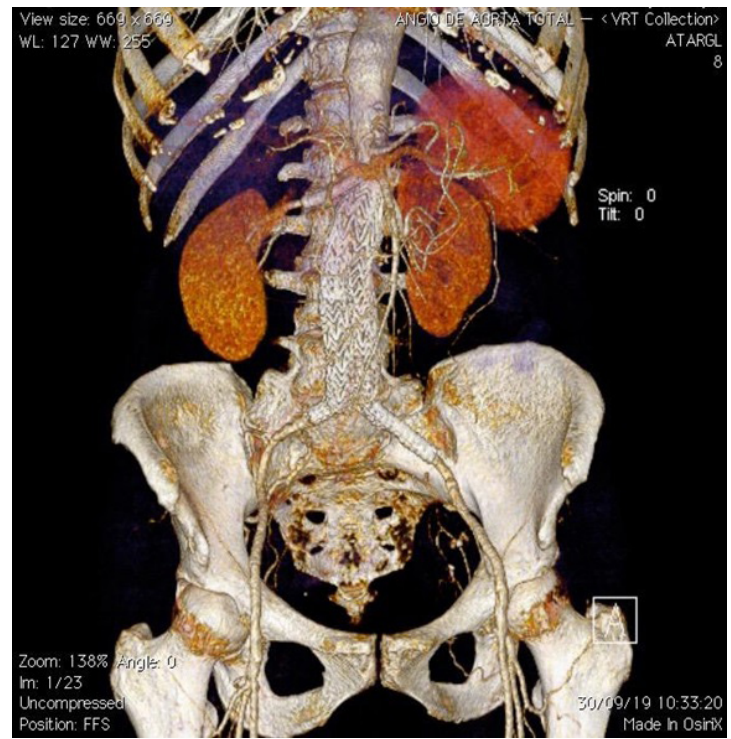

Figura 9. Angiotomografia de controle com 30 dias mostrando endoprótese e stents de artérias renais bem-posicionados, sem fraturas e sem vazamentos.

Atualmente, o TE do AAA roto é realizado nos pacientes estáveis, já que é necessária a realização prévia de uma angiotomografia que defina as medidas adequadas para esse tipo de conduta. Contudo, existem protocolos nos centros de referência com grande demanda de casos que tendem a se beneficiar do uso do REBOA (oclusão ressuscitativa por meio de balão endovascular da aorta), o que amplia e possibilita o TE para pacientes instáveis ${ }^{10}$. Vale lembrar que essa conduta não é consenso e ainda é pouco discutida no Brasil, não tendo sido realizada no nosso caso.

Nos Estados Unidos, uma análise das tendências confirmou que o EVAR está sendo cada vez mais utilizado para o tratamento dos AAA rotos, com diminuição da mortalidade associada. Também demonstrou resultados superiores quando o EVAR para aneurisma roto é realizado em hospitais de ensino e centros de alto volume. Se anatomicamente favorável, a sua opção pelo TE em detrimento da cirurgia aberta foi classificada como forte nível de recomendação (nível 1) ${ }^{11}$. No Brasil, segundo a Diretriz Brasileira para o tratamento do AAA da Comissão Nacional de Incorporação de Tecnologias no Sistema Único de Saúde (CONITEC), os AAAs rotos estáveis, com anatomia favorável ao tratamento por EVAR (confirmada pela tomografia computadorizada), devem ser tratados em caráter emergencial por cirurgia aberta ou EVAR de acordo com a experiência e disponibilidade da equipe cirúrgica e material ${ }^{2}$.

O estudo multicêntrico e randomizado IMPROVE (Immediate Management of Patients with Ruptured Aneurysm: Open Versus Endovascular Repair) 
demonstrou uma mortalidade em 30 dias semelhante para o reparo aberto $(37,4 \%)$ e endovascular (EVAR) $(35,4 \%)$. No entanto, o EVAR teve menor tempo de permanência hospitalar. Há também uma tendência de melhores resultados em mulheres ${ }^{12}$.

As endopróteses constituem uma parcela significativa dos custos do EVAR (34-52\%), mas esses custos são compensados pelo menor tempo de internação, o que difere do reparo aberto. Porém, a longo prazo, não são observadas diferenças significativas com relação ao custo, devido à necessidade de acompanhamento com imagens e reintervenções após o EVAR. Os pacientes submetidos ao EVAR geralmente têm melhor qualidade de vida relacionada à saúde nos primeiros 12 meses, embora estudos não tenham mostrado diferença significativa além do primeiro ano ${ }^{13}$.

Entre os benefícios do procedimento endovascular sobre o reparo aberto, estão a invasividade reduzida, evitando uma laparotomia, o controle vascular sob anestesia local, a estabilidade hemodinâmica, a hipotensão permissiva durante todo o procedimento e a oclusão aórtica seletiva para reduzir a hemorragia retroperitoneal. Isso pode resultar em tempo de operação mais rápido, menor perda de sangue e redução da morbidade e mortalidade cardiopulmonar perioperatória, possivelmente resultando em melhor desfecho da mortalidade em 30 dias e melhor desfecho a longo prazo ${ }^{14}$. Porém, é importante ter atenção com o tamanho do aneurisma corrigido, pois já foi relatado que, quanto maior o diâmetro do AAA, observado principalmente em casos rompidos, maior é a associação com as taxas de reintervenção ${ }^{15}$.

A técnica com stents paralelos consiste em colocar stents revestidos nas artérias viscerais, em paralelo ao corpo da endoprótese da aorta. É chamada de periscópio quando é colocado em sentido retrógrado ao fluxo sanguíneo e chamada de chaminé, snorkel e sanduíche quando colocado em sentido anterógrado ao fluxo. Normalmente, para o TE do AAA, é utilizado um oversize de 10 a $15 \%$ na endoprótese. Contudo, na técnica de chaminé, se faz necessário um oversize maior (30\%), para evitar possíveis vazamentos e goteiras ("gutters"). Também é importante um overlap (comprimento) de pelo menos $5 \mathrm{~cm}$ de stent paralelo, terminando $1 \mathrm{~cm}$ acima da endoprótese da aorta ${ }^{16}$. Não se deve usar balonamento de acomodação na aorta devido ao risco de compressão dos stents paralelos das viscerais.

A escolha pela técnica de chaminé ocorreu por diversos motivos: fácil acesso ao material necessário para o TE, experiência da equipe com essa técnica e possibilidade de tratamento em curto espaço de tempo. Além disso, há uma limitação para o uso de endoprótese fenestrada, já que o seu tempo de confecção (6-8 semanas) é elevado, pois são produzidas especificamente para cada caso. Já para as endopróteses ramificadas, a necessidade de um diâmetro mínimo de $34 \mathrm{~mm}$ impossibilitou o seu uso. Quanto às endopróteses de prateleira, ainda são disponíveis no mercado apenas com quatro ramos ou fenestras (não se adequando ao caso, no qual era necessário cobrir apenas as renais) ${ }^{17}$.

Quanto às endopróteses modificadas pelo cirurgião, é necessário ter mais experiência com relação à manipulação e modificação dos dispositivos. Além disso, há sempre o risco de contaminação do material ${ }^{18}$.

Há uma tendência crescente e evidências para apoiar o uso da técnica de chaminé nesse grupo de alto risco de pacientes, principalmente relacionadas às baixas taxas precoces de mortalidade e complicações. Resultados do estudo multicêntrico PERICLES ${ }^{19}$ (Prospective study for improvement of colonoscopy bowel preparation procedure by software supported visualization) em 2015 mostraram 517 pacientes submetidos a técnica de chaminé, com um total de 898 próteses de chaminé. O tempo médio de seguimento foi de 17 meses, e a taxa de sobrevivência em uma coorte de alto risco de pacientes foi de $79 \%$, com uma taxa primária de patência de $94 \%$ e uma taxa secundária de $95 \%$. Além disso, um estudo francês publicado em 2014 não encontrou diferença significativa nos resultados de curto e médio prazo entre pacientes tratados com a técnica de chaminé e a técnica fenestrada para o AAA justarrenal. Sendo assim, a técnica de chaminé deve ser considerada válida fora da prateleira e imediatamente disponível para tratar aneurismas complexos em um grupo de pacientes de alto risco ${ }^{19}$.

Concluímos, então, com esse caso, que o TE com a técnica de endoprótese com stents paralelos (chaminé) no AAA roto complexo se mostrou uma excelente opção terapêutica. Isso se deve principalmente à sua disponibilidade na urgência. É uma técnica factível e reprodutível, além de ser de baixo custo quando comparada às outras técnicas endovasculares. Outros estudos para avaliar o papel do TE no tratamento do AAA roto a longo prazo são necessários.

\section{REFERÊNCIAS}

1. Badger SA, Harkin DW, Blair PH, Ellis PK, Kee F, Forster R. Endovascular repair or open repair for ruptured abdominal aortic aneurysm: a Cochrane systematic review. BMJ Open. 2016;6(2):e008391. http:// dx.doi.org/10.1136/bmjopen-2015-008391. PMid:26873043.

2. Magliano CA, Senna K, Santos M. Diretriz brasileira para o tratamento do aneurisma de aorta abdominal. CONITEC. 2017;240:1-55.

3. Greenhalgh RM, Brown LC, Kwong GP, Powell JT, Thompson SG, EVAR trial participants. Comparison of endovascular aneurysm repair with open repair in patients with abdominal aortic aneurysm (EVAR trial 1), 30-day operative mortality results: randomised controlled trial. Lancet. 2004;364(9437):843-8. http://dx.doi. org/10.1016/S0140-6736(04)16979-1. PMid:15351191. 
4. EVAR trial participants. Endovascular aneurysm repair and outcome in patients unfit for open repair of abdominal aortic aneurysm (EVAR trial 2): randomised controlled trial. Lancet. 2005;365(9478):2187-92. http://dx.doi.org/10.1016/S01406736(05)66628-7. PMid:15978926.

5. Prinssen $M$, Verhoeven EL, Buth J, et al. A randomized trial comparing conventional and endovascular repair of abdominal aortic aneurysms. N Engl J Med. 2004;351(16):1607-18. http:// dx.doi.org/10.1056/NEJMoa042002. PMid:15483279.

6. Lederle FA, Freischlag JA, Kyriakides TC, et al. Long-term comparison of endovascular and open repair of abdominal aortic aneurysm. N Engl J Med. 2012;367(21):1988-97. http://dx.doi.org/10.1056/ NEJMoa1207481. PMid:23171095.

7. Aburahma AF, Campbell JE, Mousa AY, et al. Clinical outcomes for hostile versus favorable aortic neck anatomy in endovascular aortic aneurysm repair using modular devices. J Vasc Surg. 2011;54(1):13-21. http://dx.doi.org/10.1016/j.jvs.2010.12.010. PMid:21324631.

8. Faruqi RM, Chuter TA, Reilly LM, et al. Endovascular repair of abdominal aortic aneurysm using a pararenal fenestrated stent-graft. J Endovasc Surg. 1999;6(4):354-8. http://dx.doi.org/10.1583/10746218(1999)006<0354:EROAAA >2.0.CO;2. PMid:10893139.

9. Chaikof EL, Dalman RL, Eskandari MK, et al. The Society for Vascular Surgery practice guidelines on the care of patients with an abdominal aortic aneurysm. J Vasc Surg. 2018;67(1):2-77.e2. http://dx.doi.org/10.1016/j.jvs.2017.10.044. PMid:29268916.

10. Hofmeister S, Thomas MB, Paulisin J, Mouawad NJ. Endovascular management of ruptured abdominal aortic aneurysms and acute aortic dissections. Vasa. 2019;48(1):35-46. http://dx.doi. org/10.1024/0301-1526/a000760. PMid:30407131.

11. Mureebe L, Egorova N, Giacovelli JK, Gelijns A, Kent KC, McKinsey JF. National trends in the repair of ruptured abdominal aortic aneurysms.J Vasc Surg. 2008;48(5):1101-7. http://dx.doi.org/10.1016/j. jvs.2008.06.031. PMid:18771883.

12. Powell JT, Sweeting MJ, Thompson MM, et al. Endovascular or open repair strategy for ruptured abdominal aortic aneurysm: 30 day outcomes from IMPROVE randomised trial. BMJ. 2014;348:f7661. http://dx.doi.org/10.1136/bmj.f7661. PMid:24418950.

13. Kayssi A, DeBord Smith A, Roche-Nagle G, Nguyen LL. Healthrelated quality-of-life outcomes after open versus endovascular abdominal aortic aneurysm repair. J Vasc Surg. 2015;62(2):491-8. http://dx.doi.org/10.1016/j.jvs.2015.05.032. PMid:26211382.

14. Böckler D, Holden A, Krievins D, et al. Extended use of endovascular aneurysm sealing for ruptured abdominal aortic aneurysms. Semin Vasc Surg. 2016;29(3):106-13. http://dx.doi.org/10.1053/j. semvascsurg.2016.09.002. PMid:27989315.

15. Karthikesalingam A, Holt PJ, Vidal-Diez A, et al. Predicting aortic complications after endovascular aneurysm repair. $\mathrm{Br} J$ Surg. 2013;100(10):1302-11. http://dx.doi.org/10.1002/bjs.9177. PMid:23797788.

16. Lobato AC, Camacho-Lobato L. A new technique to enhance endovascular thoracoabdominal aortic aneurysm therapy-the sandwich procedure. Semin Vasc Surg. 2012;25(3):15360. http://dx.doi.org/10.1053/j.semvascsurg.2012.07.005. PMid:23062495.
17. Hu Z, Li Y, Peng R, et al. Multibranched stent-grafts for the treatment of thoracoabdominal aortic aneurysms: a systematic review and meta-analysis. J Endovasc Ther. 2016;23(4):626-33. http://dx.doi. org/10.1177/1526602816647723. PMid:27170149.

18. Kansagra K, Kang J, Taon MC, et al. Advanced endografting techniques: snorkels, chimneys, periscopes, fenestrations, and branched endografts. Cardiovasc Diagn Ther. 2018;8(Suppl 1);S17583. http://dx.doi.org/10.21037/cdt.2017.08.17. PMid:29850429.

19. Stenson KM, De Bruin JL, Holt PJ, Loftus IM, Thompson MM. Extended use of endovascular aneurysm sealing: chimneys for juxtarenal aneurysms. Semin Vasc Surg. 2016;29(3):120-5. http:// dx.doi.org/10.1053/j.semvascsurg.2016.11.006. PMid:27989317.

Correspondência Dejean Sampaio Amorim Filho Fundação Bahiana de Cardiologia Rua das Hortências, 326, Itaigara CEP 41810-010 - Salvador (BA) - Brasil Tel.: (71) 98201-3391

E-mail:dejean.a@terra.com.br

Informações sobre os autores CGA - Acadêmica do curso de Medicina, Centro Universitário FTC (UniFTC); Membro, Liga de Angiologia e Cirurgia Vascular da Bahia (LACIV).

CAPN - Especialista em Cirurgia Vascular com área de atuação em Angiorradiologia e Cirurgia Endovascular; Membro titular, Sociedade Brasileira de Angiologia e de Cirurgia Vascular (SBACV); Diretor técnico da Clínica Angiomed; Diretor de Defesa Profissional, Associação Bahiana de Medicina (ABM).

FDCA - Especialista em Cirurgia Vascular, Sociedade Brasileira de Angiologia e de Cirurgia Vascular (SBACV); Especialista em Ecografia Vascular, Colégio Brasileiro de Radiologia (CBR).

TLA - Especialista em Cirurgia Vascular com área de atuação em Angiorradiologia e Cirurgia Endovascular, Sociedade Brasileira de Angiologia e de Cirurgia Vascular (SBACV); Especialista em Ecografia Vascular pelo Colégio Brasileiro de Radiologia (CBR). FMCN - Especialista em Cirurgia Cardiovascular com área de atuação em Tratamento Endovascular das Cardiopatias Estruturais; Membro associado da Sociedade Brasileira de Angiologia e de Cirurgia Vascular (SBACV); Coordenador do grupo de Cirurgia Cardiovascular, Fundação Bahiana de Cardiologia (FBC).

DSAF - Especialista em Cirurgia Vascular com área de atuação em Angiorradiologia e Cirurgia Endovascular; Membro titular, Sociedade Brasileira de Angiologia e de Cirurgia Vascular (SBACV); Conselheiro do Conselho Regional de Medicina do Estado da Bahia

(CREMEB); Diretor administrativo da Clínica CEAVE; Diretor de Defesa Profissional da SBACV-BA; Diretor de Delegacias Regionais da Associação Bahiana de Medicina.

Contribuição dos autores Concepção e desenho do estudo: CGA, DSAF Análise e Interpretação dos dados: DSAF, CGA Coleta de dados: DSAF, CAPN, FDCA, TLA, FMCN, CGA Redação do Artigo: CGA, DSAF

Revisão crítica do texto: DSAF Aprovação final do artigo: CGA, DSAF, CAPN, FDCA, TLA, FMCN Análise estatística: N/A. Responsabilidade geral pelo estudo: DSAF, CAPN

*Todos os autores leram e aprovaram a versão final submetida ao J Vasc Bras. 